Classification

Physics Abstracts

$07.20 \mathrm{Dt}-44.10+\mathrm{i} 44.30+\mathrm{V}$

\title{
Mesure des températures de surface par contacts séparés
}

\author{
A. Gery, A. Degiovanni, D. Petit et J. Dard \\ Laboratoire de Physique Industrielle, INSA de Lyon, 69621 Villeurbanne Cedex, France
}

(Reçu le 2 juillet 1981, révisé le 27 novembre 1981, accepté le 19 janvier 1982)

\begin{abstract}
Résumé. - Les perturbations du champ de température apportées par la présence de couples appuyés sur une surface sont analysées. Un coefficient d'erreur est défini. En régime permanent, des mesures sont effectuées pour des milieux épais conducteurs ou isolants, les résultats sont en bon accord avec ceux issus d'une modélisation mettant en évidence diverses résistances thermiques notamment celles correspondant aux macro- et microconstrictions des lignes de flux. Pour des milieux fins les coefficients d'erreur sont calculés à partir du modèle. Ils peuvent atteindre des valeurs élevées : jusqu'à $60 \%$, pour des thermoéléments semiconducteurs. Il y a donc lieu d'être vigilant lors du choix des détecteurs.

En régime transitoire, des retards sont déterminés en comparant les réponses expérimentales des détecteurs et les prévisions issues d'un nouveau modèle lorsque la surface est soumise à un choc thermique. Ces retards sont faibles $(60 \mu \mathrm{s})$.

En conclusion cette étude fait apparaître l'intérêt des capteurs à contacts séparés
\end{abstract}

\begin{abstract}
When thermocouples elements are individually pressed on a surface the temperature field is modified. An error coefficient is defined. In steady state conditions, experimental investigations are done for conducting and insulating thick materials. A theoretical analysis of the heat transfer process shows thermal resistances, essentially those corresponding to the macro- and microconstriction of the heat flow near the surface. Experimental and theoretical results are similar. For thin substrate, the error coefficients are only computed from the theoretical model. The error coefficient may be very high, up to $60 \%$ for $\mathrm{Bi}_{2} \mathrm{Te}_{3}$ element, consequently care should be taken when choosing the thermocouple configuration.

In transient state delays are determined by comparing experimental measurement and temperature response resulting from a new model. Short delay times are observed $(60 \mu \mathrm{s})$.

This study shows the advantages of using thermocouples elements in contact separately with the surface.
\end{abstract}

La mesure des températures de surface par couple à contacts séparés consiste à appuyer deux thermoéléments sur le matériau; la jonction est alors assurée par le matériau lui-même s'il est conducteur électrique ou par une couche conductrice déposée lorsqu'il est isolant.

La présence des thermoéléments modifie le champ de température, cette cause d'erreur a déjà fait l'objet de nombreux travaux [1-18].

A l'échelle macroscopique, les lignes de flux, en général, convergent vers les thermoéléments, c'est l'effet de macroconstriction ; à l'échelle microscopique, le contact n'étant pas parfait il existe de nouvelles convergences vers les zones en contact réel, c'est l'effet de microconstriction ou de résistance de contact.

1. Régime permanent. - Nous voulons, ici, connaître quantitativement les améliorations apportées par ce type de détection pour diverses configurations géométriques et thermiques. Pour ce faire, nous définissons un coefficient d'erreur $\varepsilon$ différent du coefficient $K$ adopté par Cassagne, Kirsch et Bardon [4, 5]. (Il est d'ailleurs possible de montrer que, dans la plupart des cas, les valeurs issues des deux définitions se rejoignent). Le coefficient $\varepsilon$ que nous adoptons s'écrit : $\varepsilon=\frac{T-\theta}{T-T_{0}}$ où, expérimentalement $T$ représente la température de la surface extrapolée en présence des détecteurs à partir des régions situées hors de la zone de macroconstriction, $\theta$ la température indiquée par les détecteurs et $T_{0}$ une température de référence. Théoriquement, $T$ représentera la température de la surface, calculée en tenant compte d'un flux uniformément réparti sur la surface et égal à celui qui s'échapperait par les détecteurs.

1.1 DisPositif EXPÉRIMENTAL. - Nous avons mené à bien une étude expérimentale à l'aide de l'appareillage représenté sur la figure 1 . 


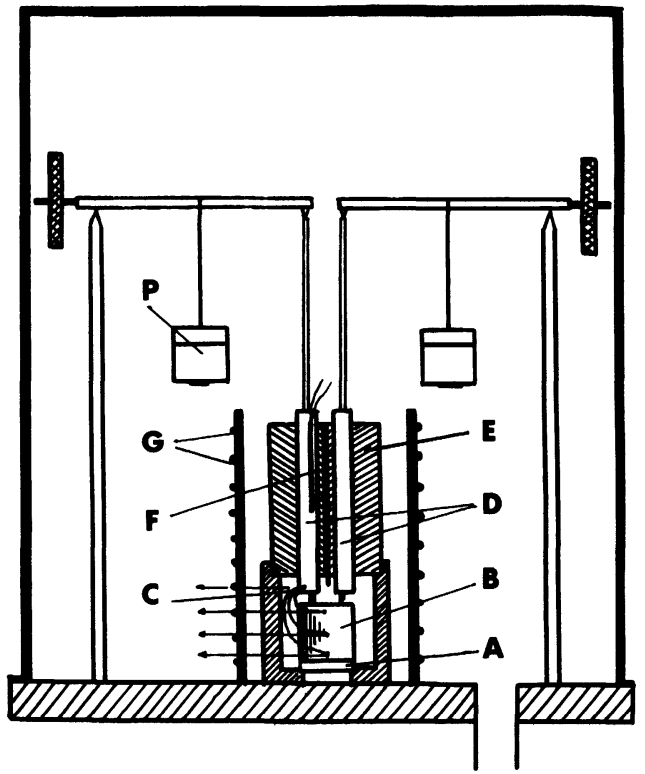

Fig. 1. - A : chaufferette; B : échantillon; C : soudure froide; D : «doigts " porteurs des éléments thermoélectriques; $\mathrm{E}$ : puits de chaleur en cuivre; $\mathrm{F}$ : sonde en platine; $G$ : circulation d'eau; $P$ : poids interchangeables.

[A : heater; B : sample; C : cold junction; D : thermoelectric element holders; $\mathrm{E}$ : copper heat sink; $\mathrm{F}$ : platinium transducer; $G$ : water circulation; $P$ : weight.]

Avec ce dispositif, il est possible de faire varier :

- la force d'application des détecteurs sur la surface et la pression gazeuse du milieu ambiant,

- la conductivité thermique du milieu,

- la forme et la nature des détecteurs,

- l'épaisseur du dépôt superficiel métallique nécessaire pour fermer le circuit électrique du couple lorsque le milieu est isolant.

Les échantillons sont cylindriques, de diamètre $2 \mathrm{~cm}$ et de hauteur $3 \mathrm{~cm}$. Les trois thermocouples permettant d'extrapoler la température en surface sont en nickelchrome, nickel allié de $8 \times 10^{-5} \mathrm{~m}$ de diamètre. Ils sont disposés comme l'indique la figure 2 .

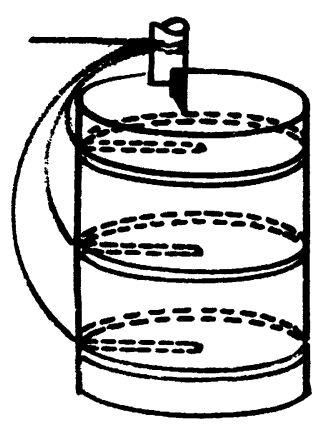

Fig. 2. - Montage des relevés de température direct et indirect.

[Direct and indirect surface temperature measurement.]
La température de l'échantillon est élevée au-dessus de la température environnante à l'aide d'une chaufferette de puissance $0,7 \mathrm{~W}$; dans ces conditions l'élévation de température extrapolée à la surface au-dessus de la température environnante est comprise entre 10 et $20^{\circ} \mathrm{C}$ suivant les échantillons. Pour les matériaux les plus isolants l'écart de température entre les thermocouples externes est de quelques degrés.

La géométrie des couples testés est représentée sur les schémas 3 et 4.

Le flux quittant l'échantillon par les thermocouples appuyés est très faible; nous avons en effet, vérifié expérimentalement que les températures extrapolées en présence ou en absence de ces thermocouples étaient quasiment identiques.

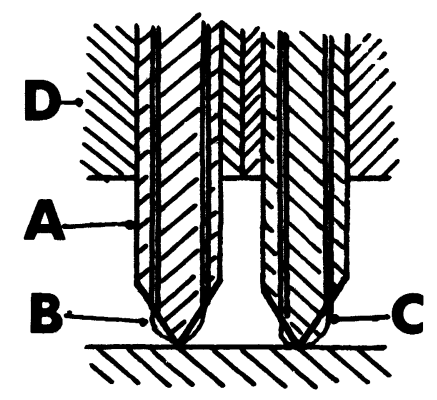

Fig. 3. - A : gaine d'alumine bifilaire $\varnothing: 1,2 \times 10^{-3} \mathrm{~m}$; B : nickel-chrome $\varnothing: 8 \times 10^{-5} \mathrm{~m} ; \mathrm{C}$ : nickel allié $\varnothing$ : $8 \times 10^{-5} \mathrm{~m} ; \mathrm{D}:$ cylindre de cuivre.

[A : insulator bifilar shield (diameter : $1.2 \times 10^{-3} \mathrm{~m}$ ); B : Ni-Cr thermocouple wire (diameter : $8 \times 10^{-5} \mathrm{~m}$ ); $\mathrm{C}: \mathrm{Ni}-\mathrm{Al}$ thermocouple wire (diameter : $8 \times 10^{-5} \mathrm{~m}$ ); D : copper cylinder.]

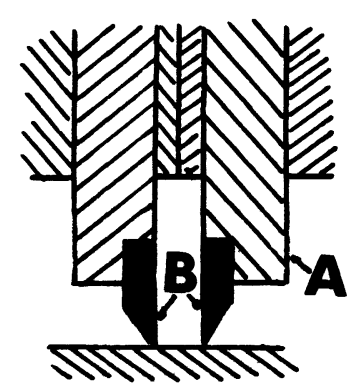

Fig. 4. - A : cylindre de cuivre; $\mathrm{B}$ : plaquettes en $\mathrm{Bi}_{2} \mathrm{Te}_{3}$ dopées $\mathbf{P}$ et $\mathbf{N}$.

[A : copper cylinder; $\mathrm{B}: \mathrm{Bi}_{2} \mathrm{Te}_{3}$ doped wafer (type $\mathrm{n}$ and p).]

1.2 MilieuX ISOlants ÉPAis. - Nous avons, avec l'appareillage précédemment décrit, effectué des mesures sur des échantillons isolants, d'une part à épaisseur de dépôt égale $\left(10^{-7} \mathrm{~m}\right)$ pour des conductivités de milieux $\left(\lambda_{3}\right)$ différentes, d'autre part, à $\lambda_{3}$ égale, pour des épaisseurs de dépôts croissantes. En effet, la couche métallique superficielle, par sa présence 
même, change l'état de surface donc risque de modifier notamment sa dureté(donc la valeur de la résistance de contact). De plus, par son épaisseur, elle doit influencer le « taux » de macroconstriction. Enfin, elle intervient

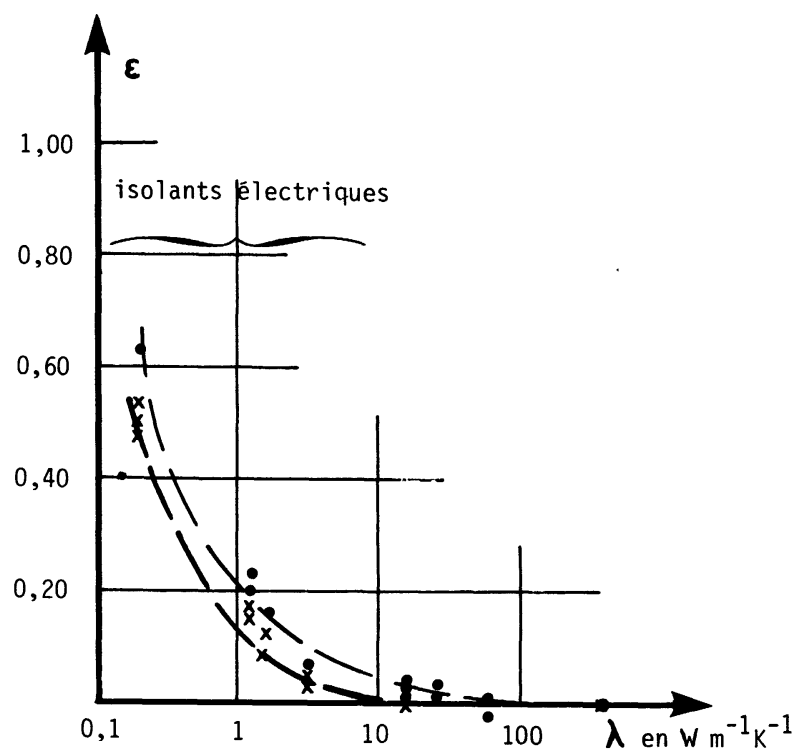

Fig. 5. - Détecteurs en $\mathrm{Bi}_{2} \mathrm{Te}_{3}$; contacts séparés. • Points expérimentaux avec : pression d'air $5 \times 10^{-3}$ torr; forces d'appui : 1,$3 ; 3,8$ et $6,6 \mathrm{~N}$. $\times$ Points expérimentaux avec : pression d'air 760 torr; forces d'appui : 1,$3 ; 3,8$ et $6,6 \mathrm{~N}$.

$\left[\mathrm{Bi}_{2} \mathrm{Te}_{3}\right.$ elements; separated contact. Experimental points : air pressure $5 \times 10^{-3}$ torr; load $1.3 ; 3.8 ; 6.6 \mathrm{~N}$. $\times$ Experimental points : air pressure 760 torr; load 1.3; $3.8 ; 6.6 \mathrm{~N}$.]

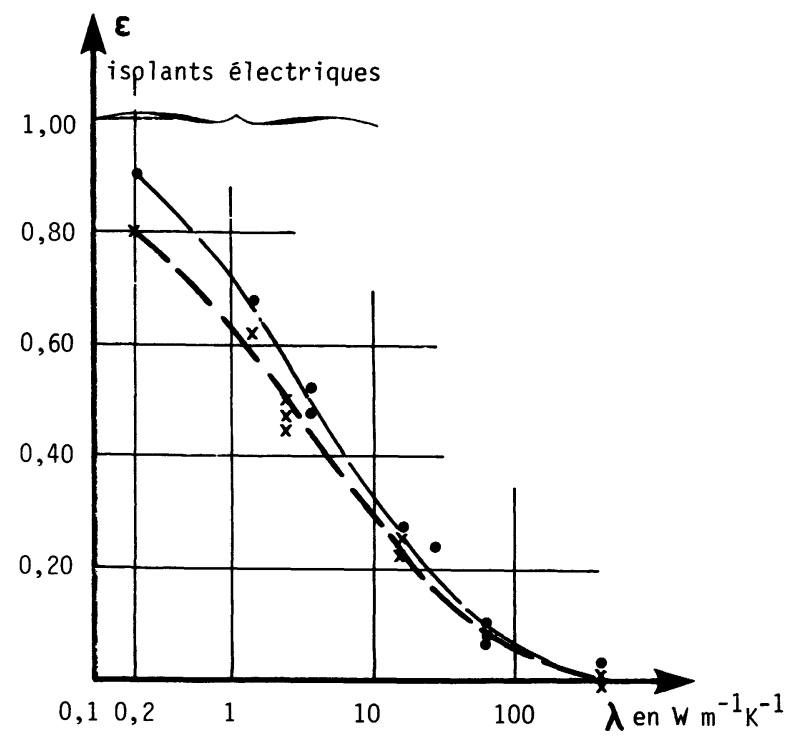

Fig. 6. - Fils détecteurs en nickel-chrome-nickel allié de diamètre $0,8 \mathrm{~mm}$; contacts séparés. • Points expérimentaux avec : pression d'air $5 \times 10^{-3}$ torr; forces d'appui : 1,3; 3,8 et $6,6 \mathrm{~N}$. $\times$ Points expérimentaux avec pression d'air 760 torr; forces d'appui : 1,$3 ; 3,8$ et $6,6 \mathrm{~N}$.

[Ni-Cr, Ni-Al elements, separated contact (diameter : $0.8 \mathrm{~mm}$ ). $\bullet$ Experimental points : air pressure $5 \times 10^{-3}$ torr ; load $1.3 ; 3.8 ; 6.6 \mathrm{~N}$. $\times$ Experimental points : air pressure 760 torr ; load $1.3 ; 3.8 ; 6.6 \mathrm{~N}$.] également sur le niveau exact de la jonction, donc sur la température $\theta$ fournie par le couple. Les résultats principaux sont portés respectivement sur les courbes 5 , 6,7 et 8 . L'évolution, en fonction de $\lambda_{3}$, du coefficient

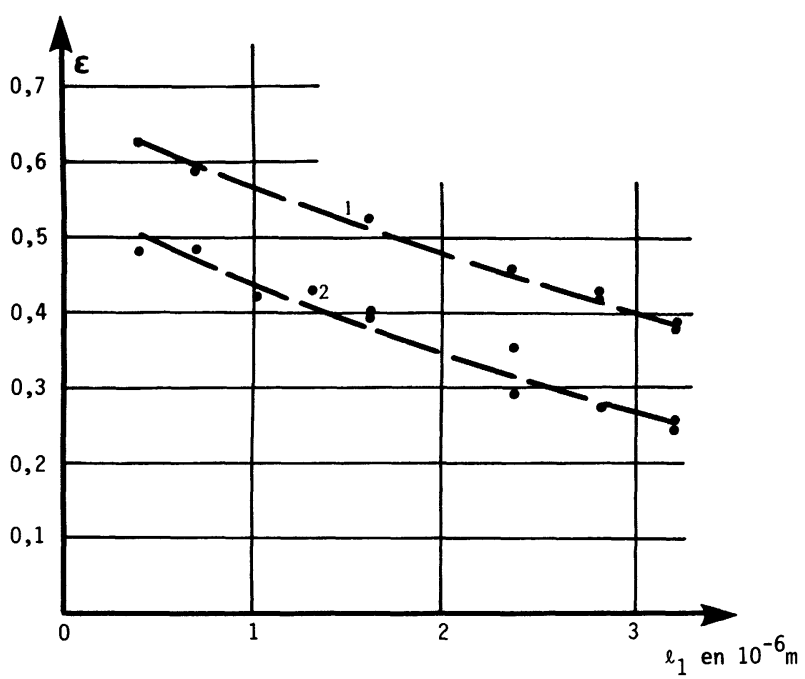

Fig. 7. - Variations du coefficient $\varepsilon$ en fonction de la couche superficielle d'or déposée par vaporisation sous vide sur plexiglass. Détecteurs en $\mathrm{Bi}_{2} \mathrm{Te}_{3}$. $\bullet$ Points expérimentaux correspondant : 1 : à une pression d'air de $10^{-2}$ torr et une force d'appui de $1,3 \mathrm{~N} ; 2$ : à la pression atmosphérique et une force d'appui de $6,6 \mathrm{~N}$.

[Error coefficient $\varepsilon$ versus the thickness of a vacuum gold deposited thin film. $\mathrm{Bi}_{2} \mathrm{Te}_{3}$ elements. $\bullet$ Experimental points : 1 : air pressure $10^{-2}$ torr; load $1.3 \mathrm{~N} ; 2$ : air pressure 760 torr; load $6.6 \mathrm{~N}$.]

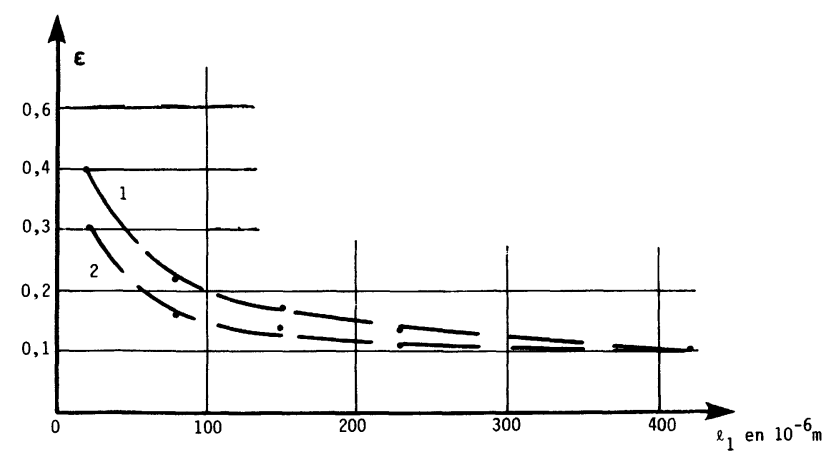

Fig. 8. - Variations du coefficient $\varepsilon$ en fonction de l'épaisseur d'une couche superficielle de laque d'or déposée au pinceau sur plexiglass. Détecteurs en $\mathrm{Bi}_{2} \mathrm{Te}_{3}$. - Points expérimentaux. 1 : pression d'air $5 \times 10^{-3}$ torr; force d'appui $1,3 \mathrm{~N} ; 2$ : pression atmosphérique; force d'appui $6,6 \mathrm{~N}$.

[Error coefficient $\varepsilon$ versus the thickness of a brush deposited gold point on a perspex sample. $\mathrm{Bi}_{2} \mathrm{Te}_{3}$ elements. Experimental points : 1 : air pressure $5 \times 10^{-3}$ torr; load $1.3 \mathrm{~N}$; 2 : air pressure 760 torr; load $6.6 \mathrm{~N}$.] 
d'erreur pour des dépôts métalliques très minces semble liée à celle de la conductivité de l'échantillon lui-même. Par contre, il faut noter qu'en faisant croître de façon "considérable " l'épaisseur d'une couche métallique conductrice thermique (jusqu'à $400 \mu \mathrm{m}$ ) le coefficient $\varepsilon$ diminue nettement et tend vers celui qui serait obtenu avec un milieu de conductivité thermique égal à celui du dépôt (courbe 8).

Nous avons cherché à prévoir théoriquement ces résultats et à mettre en valeur le rôle des divers paramètres en modélisant le problème.

La géométrie et les caractéristiques du modèle sont indiquées ci-dessous.

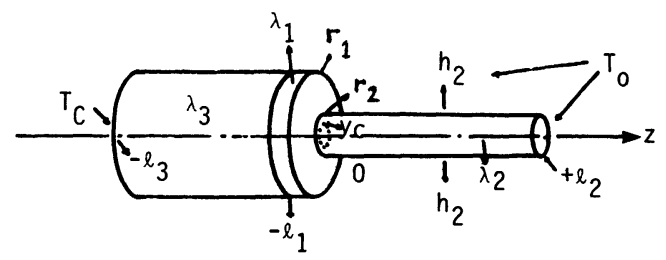

$T_{\mathrm{c}}:$ température imposée en $-l_{3}$,

$\phi$ : densité de flux traversant le contact sur un rayon de contact apparent $y_{\mathrm{c}}$

$\lambda_{3}$ : conductivité du milieu 3 , de rayon $r_{1}$ et d'épaisseur $l_{3}$,

$\lambda_{1}$ : conductivité du dépôt superficiel,

$\lambda_{2}$ : conductivité du milieu 2, détecteur de rayon $r_{2}$ et de longueur $l_{2}$, celui-ci échange de la chaleur avec une ambiance à la température $T_{0}$ (coefficient d'échange $h_{2}$ sur son pourtour et température fixée en $z=+l_{2}$ ).

Fig. 9. - Géométrie du modèle mathématique.

[Geometry of the mathematical model.]

Ces données se traduisent mathématiquement par le système suivant :

(milieu 3) $\left\{\begin{array}{l}\Delta T_{3}(z, r)=0 \\ T_{3}\left(-l_{3}, r\right)=T_{c} \\ \frac{\partial T_{3}\left(z, r_{1}\right)}{\partial r}=0\end{array}\right.$ (milieu 1) $\left\{\begin{array}{l}\frac{\Delta T_{1}(z, r)=0}{\frac{\partial T_{1}(0, r)}{\partial z}=-\frac{\phi}{\lambda_{1}}} \text { pour } \quad 0<r \leqslant y_{c} \\ \frac{\partial T_{1}(0, r)}{\partial z}=0 \quad \text { pour } \quad y_{c}<r \leqslant r_{1} \\ \frac{\partial T_{1}\left(z, r_{1}\right)}{\partial r}=0\end{array}\right.$

(milieu 2) $\left\{\begin{array}{l}\Delta T_{2}=0 \\ \frac{\partial T_{2}(0, r)}{\partial z}=-\frac{\phi}{\lambda_{2}} \text { pour } 0<r \leqslant y_{\mathrm{c}} \\ \frac{\partial T_{2}(0, r)}{\partial z}=0 \quad \text { pour } y_{\mathrm{c}}<r \leqslant r_{2} \\ T_{2}\left(l_{2}, r\right)=T_{0} \\ \lambda_{2} \frac{\partial T_{2}\left(z, r_{2}\right)}{\partial r}=-h_{2}\left[T_{2}\left(z, r_{2}\right)-T_{0}\right] .\end{array}\right.$

Passage du milieu 3 au milieu 1 :

$$
\begin{aligned}
\lambda_{1} \frac{\partial T_{1}\left(-l_{1}, r\right)}{\partial z} & =\lambda_{3} \frac{\partial T_{3}\left(-l_{1}, r\right)}{\partial z} \\
T_{1}\left(-l_{1}, r\right) & =T_{3}\left(-l_{1}, r\right) .
\end{aligned}
$$

Passage du milieu 1 au milieu 2 :

$$
\bar{T}_{1}(0, r)-\bar{T}_{2}(0, r)=r_{\mathrm{c}} \phi
$$

où $r_{\mathrm{c}}$ est une résistance de contact par unité de surface de contact et les $\bar{T}_{\mathrm{i}}$ sont des températures moyennes par rapport à la surface d'échange.

Les hypothèses thermiques choisies pour le modèle sont liées au but recherché, à savoir : comparer des niveaux de températures avec et sans détecteur. En particulier, si la surface totale échangeait avec l'extérieur cela reviendrait à superposer une densité de flux $\phi^{\prime}$ à celle, $\phi$, traversant le contact. Ceci n'entraînerait que peu de modifications pour la surface cachée ou perturbée par le détecteur étant donné la faible valeur du rapport surface-détecteur sur surface totale.

Après résolution par la méthode de séparation des variables, la solution s'écrit :

$$
T_{\mathrm{c}}-T_{0}=\phi\left[r_{\mathrm{c}}+F_{30}+F_{10}+\sum_{n=1}^{\infty} F_{1 n}+\sum_{n=1}^{\infty} F_{2 n}\right]
$$

avec

$$
\begin{gathered}
F_{30}=\frac{r_{1}}{\lambda_{3}} y_{\mathrm{c}}^{*}\left(l_{3}^{*}-l_{1}^{*}\right) ; \quad F_{10}=\frac{r_{1}}{\lambda_{1}} y_{\mathrm{c}}^{*} l_{1}^{*} ; \\
\sum_{n} F_{1 n}=\frac{4 r_{1}}{\lambda_{1}} \sum_{n=1}^{\infty} \frac{y_{\mathrm{c}}^{*} J_{1}\left(\omega_{n} y_{\mathrm{c}}^{*}\right)}{\omega_{n}^{3} J_{0}^{2}\left(\omega_{n}\right)} \frac{U_{n}+V_{n}}{V_{n}-U_{n}} \\
\sum_{n} F_{2 n}=\frac{4 r_{2}}{\lambda_{2}} \sum_{n=1}^{\infty} \frac{J_{1}^{2}\left(\alpha_{n}^{*} y_{\mathrm{c}}^{*}\right) \operatorname{th}\left(\alpha_{n}^{*} l_{2}^{*}\right)}{\alpha_{n}^{* 3} J_{1}^{2}\left(\alpha_{n}^{*}\right)\left[\frac{\alpha_{n}^{* 2}}{H_{2}^{2}}+1\right]}
\end{gathered}
$$


expressions dans lesquelles

$$
\begin{aligned}
& U_{n}=\frac{\lambda_{1}+\lambda_{3}}{2 \lambda_{1}}+\exp 2 \omega_{n}\left(l_{3}^{*}-l_{1}^{*}\right) \frac{\lambda_{3}-\lambda_{1}}{2 \lambda_{1}} \\
& V_{n}=\frac{\lambda_{1}-\lambda_{3}}{2 \lambda_{1}} \exp 2 \omega_{n} l_{1}^{*}-\frac{\lambda_{3}+\lambda_{1}}{2 \lambda_{1}} \exp 2 \omega_{n} l_{3}^{*} \\
& r_{2}^{*}=\frac{r_{2}}{r_{1}}, \quad l_{3}^{*}=\frac{l_{3}}{r_{1}}, \quad l_{1}^{*}=\frac{l_{1}}{r_{1}}, \quad J_{1}\left(\omega_{n}\right)=0 \\
& l_{2}^{*}=\frac{l_{2}}{r_{1}}, \quad r_{1}^{*}=\frac{r_{1}}{r_{1}}=1, \quad H_{2}=\frac{h_{2} r_{1}}{\lambda_{2}} \quad \text { et } \quad \alpha_{n} r_{1}=\alpha_{n}^{*} \quad J_{0}\left(\alpha_{n}^{*}\right)=\frac{\alpha_{n}^{*}}{H_{2}} J_{1}\left(\alpha_{n}^{*}\right)
\end{aligned}
$$

La relation (1) peut s'écrire en termes de résistances thermiques :

$T_{\mathrm{c}}-T_{0}=\Phi\left[R_{\mathrm{c}}+R_{\mathrm{th} 3}+R_{\mathrm{th} 1}+R_{\mathrm{M}}+R_{\mathrm{D}}\right] \quad$ avec

$R_{\mathrm{c}} \quad$ résistance totale de contact $=\frac{r_{\mathrm{c}}}{\pi y_{\mathrm{c}}^{2}}$;

$R_{\mathrm{th} 3}$ résistance thermique du milieu $3=\frac{\sum F_{30}}{\pi y_{\mathrm{c}}^{2}}=\frac{r_{\mathrm{th} 3}}{\pi y_{\mathrm{c}}^{2}}$;

$R_{\mathrm{th} 1}$ résistance thermique du milieu $1=\frac{\sum F_{10}}{\pi y_{\mathrm{c}}^{2}}=\frac{r_{\mathrm{th} 1}}{\pi y_{\mathrm{c}}^{2}}$

$R_{\mathrm{M}}$ résistance de macroconstriction $=\frac{\sum F_{1 n}}{\pi y_{\mathrm{c}}^{2}}=\frac{r_{\mathrm{m}}}{\pi y_{\mathrm{c}}^{2}} ;$

$R_{\mathrm{D}}$ résistance thermique du détecteur $=\frac{\sum F_{2 n}}{\pi y_{\mathrm{c}}^{2}}=\frac{r_{\mathrm{D}}}{\pi y_{\mathrm{c}}^{2}}$.

Le coefficient d'erreur $\varepsilon=\frac{T-\theta}{\theta-T_{0}}$ est calculable sachant que

$$
T=T_{\mathrm{c}}-\left(r_{\mathrm{th} 3}+r_{\mathrm{th} 1}\right) \phi
$$

et

$$
\frac{\bar{T}_{1}-\theta}{\theta-T_{2}}=\frac{r_{\mathrm{c} 1}}{r_{\mathrm{c} 2}}
$$

où $r_{\mathrm{c} 1}$ est la résistance de contact par unité de surface du côté du milieu 1 et $r_{\mathrm{c}}$ la résistance totale de contact $\left(r_{\mathrm{c}}=r_{\mathrm{c} 1}+r_{\mathrm{c} 2}\right)$.

On peut ainsi écrire :

$$
\varepsilon=\frac{r_{\mathrm{c} 1}+r_{\mathrm{m}}}{r_{\mathrm{c}}+r_{\mathrm{m}}+r_{\mathrm{D}}}
$$

$r_{\mathrm{c}}$ et $r_{\mathrm{c} 1}$ sont calculées indépendamment avec un modèle et des hypothèses similaires à celles du modèle précédent (8). Il suffit de signaler que la même série $\sum F_{1 n}$ et sa limite (appelée $\sum F_{1 n}^{\prime}$ ) quand $l_{1} \Rightarrow 0$, interviennent dans la solution. Ces séries permettent donc de définir aussi bien la résistance de macroconstriction que la résistance de contact. L'évolution de ces séries, symbolisant celle des résistances thermiques, est représentée sur l'exemple d'abaques 10.

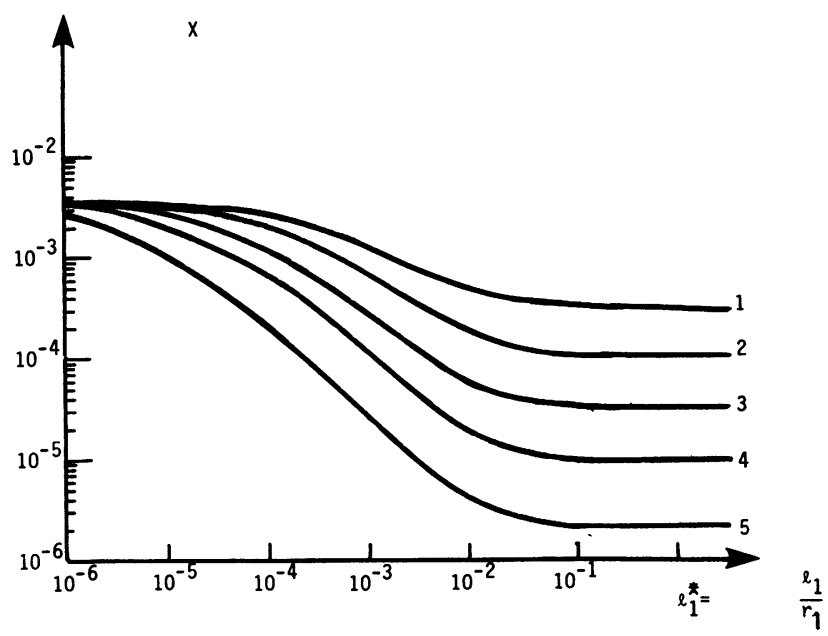

Fig. 10. - Exemple d'abaque avec : $r_{1}$ rayon de l'échantillon; $y_{c}$ rayon de contact apparent.

\begin{tabular}{c|c|c|c|c|c|c|}
\hline Courbes & $\mathrm{N} 0$ & 1 & 2 & 3 & 4 & 5 \\
\hline & $\frac{\lambda_{3}}{\lambda_{1}}$ & 0,1 & $0,34 \times 10^{-1}$ & $0,1 \times 10^{-1}$ & $0,34 \times 10^{-2}$ & $0,68 \times 10^{-3}$ \\
$r_{\mathrm{m}}=X \frac{r_{1}}{\lambda_{3}}$.
\end{tabular}

[Example of graph : $r_{1}$ sample radius; $y_{\mathrm{c}}$ apparent radius contact.] 
En fonction de $\lambda_{3} / \lambda_{1}$ (rapport des conductivités du milieu isolant et du dépôt), des rapports de rayons relatifs aux calculs des résistances de macroconstriction et microconstriction (pour la macroconstriction $r_{1} / r_{2}$ et pour la microconstriction du rapport $\mathrm{du}$ rayon de contact vrai au rayon de la cellule de microconstriction) et de l'épaisseur de la couche, ces abaques confirment l'expérience :

- si l'épaisseur $l_{1}$ du dépôt est très faible, les séries tendent vers une valeur fixe égale à celle correspondant à une absence de couche,

- si l'épaisseur $l_{1}$ du dépôt croît, un palier inférieur est également atteint.

Enfin si le rapport $\lambda_{3} / \lambda_{1}$ décroît, les résistances $R_{\mathrm{M}}$ et $R_{\mathrm{c}}$ (macro et micro-constriction) décroissent.

1.3 MilieUX Conducteurs ÉPAis. - Pour ces milieu, les essais effectués nous ont donné les résultats consignés dans les tableaux I et II :

Tableau $\mathrm{I} .-\varepsilon$ pour des détecteurs en $\mathrm{Bi}_{2} \mathrm{Te}_{3}$ à contacts séparés $\left(\lambda_{2}=1 \mathrm{~W} \cdot \mathrm{m}^{-1} \cdot \mathrm{K}^{-1}\right)$.

\begin{tabular}{|c|c|c|c|c|c|c|c|}
\hline & Forces en newtons & 1,3 & & 3,8 & & 6,6 & \\
\hline & Pression en $\mathrm{mmHg}$ & $5 \times 10^{-3}$ & 760 & $5 \times 10^{-3}$ & 760 & $5 \times 10^{-3}$ & 760 \\
\hline $\begin{array}{l}\lambda_{3} \text { en } \mathrm{W} \cdot \mathrm{m}^{-1} \cdot \mathrm{K}^{-1} \\
\text { conductivité des milieux }\end{array}$ & $\begin{array}{r}390 \\
60 \\
16\end{array}$ & $\begin{array}{l}0 \\
0,01 \\
0,05\end{array}$ & $\begin{array}{l}0 \\
0 \\
0\end{array}$ & $\begin{array}{l}0,01 \\
0,02 \\
0,04\end{array}$ & $\begin{array}{l}0 \\
0 \\
0\end{array}$ & $\begin{array}{l}0,01 \\
0,02 \\
0,02\end{array}$ & $\begin{array}{l}0 \\
0 \\
0\end{array}$ \\
\hline
\end{tabular}

Tableau II. $-\varepsilon$ pour des détecteurs en nickel-chrome-nickel allié sous gaine d'alumine, à contacts séparés $\left(\lambda_{2}\right.$ moyen $\left.=15 \mathrm{~W} \cdot \mathrm{m}^{-1} \cdot \mathrm{K}^{-1}\right)$.

\begin{tabular}{|l|c|cc|cc|cc|}
\hline & Forces en newtons & 1,3 & 3,8 & \multicolumn{2}{|c|}{6,6} \\
\cline { 2 - 7 } & Pression en mmHg & $5 \times 10^{-3}$ & 760 & $5 \times 10^{-3}$ & 760 & $5 \times 10^{-3}$ & 760 \\
\hline & 390 & 0,04 & 0,01 & 0,04 & 0,01 & 0,01 & 0 \\
$\lambda_{3}$ en $\mathbf{W} \cdot \mathrm{m}^{-1} \cdot \mathrm{K}^{-1}$ & 60 & 0,10 & 0,09 & 0,08 & 0,09 & 0,07 & 0,09 \\
conductivité des milieux & 16 & 0,28 & 0,26 & 0,28 & 0,23 & 0,28 & 0,26 \\
\hline
\end{tabular}

On constate que $\varepsilon$ est d'autant plus faible que le rapport des conductivités du milieu et du thermomètre est grand $\left(\lambda_{3} / \lambda_{2}\right)$.

Par contre l'influence de la force d'appui et de la pression gazeuse ambiante n'apparaît pas de façon très significative.

Nous avons retrouvé ces considérations à partir du modèle précédent avec

$$
l_{1}=0 \quad \text { et } \quad r_{\mathrm{c} 3}=\frac{\lambda_{2}}{\lambda_{2}+\lambda_{3}} .
$$

La température $\theta$ est alors définie par l'expression :

$$
\frac{\bar{T}_{3}-\theta}{\theta-\bar{T}_{2}}=\frac{\lambda}{\lambda_{3}} \text { et } \varepsilon \text { est égal à } \frac{r_{\mathrm{m}}+r_{\mathrm{c} 3}}{r_{\mathrm{m}}+r_{\mathrm{D}}+r_{\mathrm{c}}} \text {. }
$$

Les calculs numériques effectués avec cette modélisation permettent de retrouver les résultats expérimentaux de façon correcte.

La conclusion la plus remarquable à tirer des deux études (théorique et expérimentale) est le rôle joué par le rapport des conductivités $\lambda_{3} / \lambda_{2}$ sur le niveau de température $\theta$ mesuré.
Le rôle de ces conductivités montre clairement l'intérêt des contacts séparés par rapport à un détecteur monocontact sur l'expression du coefficient d'erreur :

$$
\varepsilon=\frac{R_{\mathrm{m}}+R_{\mathrm{c}} \frac{\lambda_{2}}{\lambda_{2}+\lambda_{3}}}{R_{\mathrm{m}}+R_{\mathrm{c}}+R_{\mathrm{D}}} \text { (contacts séparés) }
$$

toujours inférieur à la forme :

$$
K^{\prime}=\frac{R_{\mathrm{c}}+R_{\mathrm{c}}}{R_{\mathrm{m}}+R_{\mathrm{c}}+R_{\mathrm{D}}} \text { (monocontact). }
$$

L'erreur, pour des contacts séparés, sera d'autant plus faible que la conductivité du détecteur sera peu élevée. Les détecteurs en $\mathrm{Bi}_{2} \mathrm{Te}_{3}$ possédant une conductivité de $1 \mathrm{~W} \cdot \mathrm{m}^{-1} \cdot \mathrm{K}^{-1}$ sont de ce point de vue particulièrement performants.

1.4 MilieuX Fins. - Etant donné l'impossibilité de placer des thermocouples dans un milieu de faible épaisseur pour pouvoir extrapoler la température de surface, nous nous bornons à calculer les coefficients $\varepsilon$ à partir des modèles. 
Pour des milieux suffisamment épais, une condition de flux ou de température imposée en $z=-l_{3}$ conduit aux mêmes résultats. Par contre, pour les milieux fins, cette condition influe énormément sur la forme des constrictions qu'elle impose. Les exemples du tableau III où nous donnons tous les termes servant aux calculs de $\varepsilon$, pour les deux conditions : flux ou température imposée en $z=-l_{3}$, le prouvent :

Tableau III

\begin{tabular}{|c|c|c|}
\hline$\left(\pi r_{1}^{2}=4,5 \times 10^{-6} \mathrm{~m}\right)$ & \multicolumn{2}{|c|}{$\begin{array}{l}\text { Epaisseur du milieu } l_{3}=10^{-4} \mathrm{~m} \\
\text { conductivité des détecteurs } \lambda_{2}=1 \mathrm{~W} \cdot \mathrm{m}^{-1} \cdot \mathrm{K}^{-1} \\
\text { contacts séparés } \\
\qquad R_{\mathrm{D}}=1800 \mathrm{~K} \cdot \mathrm{W}^{-1}\end{array}$} \\
\hline \multirow{3}{*}{$\lambda_{3}=0,2 \mathrm{~W} \cdot \mathrm{m}^{-1} \cdot \mathrm{K}^{-1}$} & \multicolumn{2}{|c|}{$R_{\mathrm{c} 3}=185 \mathrm{~K} \cdot \mathrm{W}^{-1}\left({ }^{*}\right)$} \\
\hline & Température imposée en $-l_{3}$ & Flux imposé en $-l_{3}$ \\
\hline & $\begin{array}{l}R_{\mathrm{M}}=100 \mathrm{~K} \cdot \mathrm{W}^{-1} \\
\varepsilon \quad=0,13\end{array}$ & $\begin{aligned} R_{\mathrm{M}} & =17800 \mathrm{~K} \cdot \mathrm{W}^{-1} \\
\varepsilon & =0,91\end{aligned}$ \\
\hline \multirow{3}{*}{$\lambda_{3}=1 \mathrm{~W} \cdot \mathrm{m}^{-1} \cdot \mathrm{K}^{-1}$} & \multicolumn{2}{|c|}{$R_{\mathrm{c} 3}=55 \mathrm{~K} \cdot \mathrm{W}^{-1}$} \\
\hline & Température imposée en $-l_{3}$ & Flux imposé en $-l_{3}$ \\
\hline & $\begin{array}{l}R_{\mathrm{M}}=21 \mathrm{~K} \cdot \mathrm{W}^{-1} \\
\varepsilon=0,039\end{array}$ & $\begin{aligned} R_{\mathrm{M}} & =3500 \mathrm{~K} \cdot \mathrm{W}^{-1} \\
\varepsilon & =0,66\end{aligned}$ \\
\hline \multirow{3}{*}{$\lambda_{3}=100 \mathrm{~W} \cdot \mathrm{m}^{-1} \cdot \mathrm{K}^{-1}$} & \multicolumn{2}{|c|}{$R_{\mathrm{c}}=90 \mathrm{~K} \cdot \mathrm{W}^{-1} \quad R_{\mathrm{c} 3}=0,9 \mathrm{~K} \cdot \mathrm{W}^{-1}$} \\
\hline & Température imposée en $-l_{3}$ & Flux imposé en $-l_{3}$ \\
\hline & $\begin{array}{l}R_{\mathrm{M}}=0,21 \mathrm{~K} \cdot \mathrm{W}^{-1} \\
\varepsilon=0,0006\end{array}$ & $\begin{array}{l}R_{\mathrm{M}}=31 \mathrm{~K} \cdot \mathrm{W}^{-1} \\
\varepsilon=0,019\end{array}$ \\
\hline
\end{tabular}

$\left(^{*}\right)$ Nous avons supposé que, même pour ces milieux fins, la relation $R_{\mathrm{c} 3}=R_{\mathrm{c}} \frac{\lambda_{2}}{\lambda_{2}+\lambda_{3}}$ était encore valable.

On voit nettement que les variations de résistance de macroconstriction entraînent de gros écarts sur les coefficients d'erreur pour des milieux de faible épaisseur.

1.5 Conclusion. - Pour toutes les configurations étudiées, l'intérêt des contacts séparés en tellurure de bismuth est mis en valeur pour des raisons diverses. Pour les milieux épais et conducteurs, le niveau de température de la jonction proche de la température de la surface en est la cause principale. Pour les milieux épais et isolants, la diminution de la résistance de macroconstriction occasionnée par la présence d'une couche superficielle métallique apparaît comme le phénomène le plus important.

Nous rejoignons ici, les conclusions de Bardon [4] sur l'intérêt d'un disque conducteur en surface, même dans le cas d'un thermomètre monocontact, pour diminuer le coefficient d'erreur. Un compromis doit être adopté quant à la dimension de ce disque pour qu'il perturbe le moins possible la surface tout en diminuant la macroconstriction : un rayon égal environ à 10 fois celui du thermomètre semble être une dimension raisonnable.

Enfin si l'on adopte une méthode de mesure à contacts séparés, on ne peut que conseiller fortement l'emploi de détecteurs semi-conducteurs; leurs caractéristiques sont bénéfiques. De plus, pratiquement, ils sont faciles à mettre en ouvre et théoriquement, étant massifs, ils sont aisément modélisables, ce qui permet de prévoir leurs performances.

2. Régime transitoire. - Dans cette partie, nous évaluons les retards essentiellement introduits dans la mesure par des détecteurs thermoélectriques $\left(\mathrm{Bi}_{2} \mathrm{Te}_{3}\right)$ appuyés séparément sur une surface $[6,8]$, lorsque cette dernière subit une variation brutale de température.

Après avoir décrit le modèle thermocinétique qui sert de base théorique à cette étude, nous traiterons successivement :

- un milieu "épais " recevant un Dirac de flux uniformément réparti sur la face opposée aux détecteurs (résultats obtenus uniquement à partir du modèle);

- un milieu « fin " recevant un flux bref uniformément réparti sur la face opposée aux détecteurs (résultats expérimentaux comparés à ceux fournis par le modèle); 
- un modèle "épais" recevant un flux bref uniformément réparti sur la face où sont appuyés

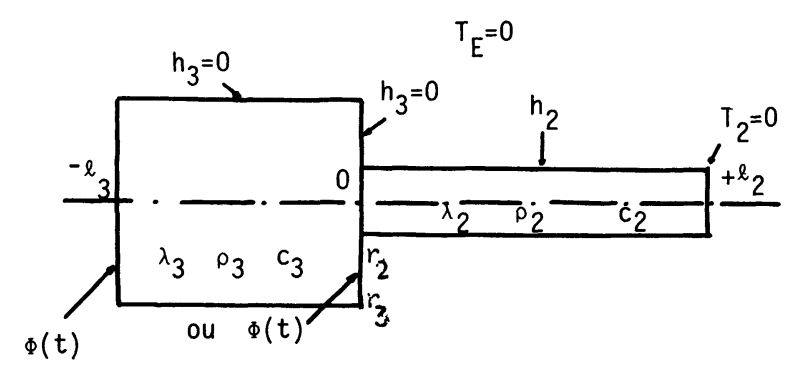

Fig. 11. - Modèle thermocinétique.

[Thermal model.] les détecteurs (résultats expérimentaux comparés à ceux fournis par le modèle).

2.1 MODÈLE THERMOCINÉTIQUE. - La géométrie du modèle choisi est représentée sur la figure 11 .

La nomenclature des données géométriques et thermiques est fournie ci-après :

$\phi(t)$ ou $\phi^{\prime}(t)$ densités de flux absorbé, indépendant de $r$,

$\lambda_{\mathbf{i}}$ conductivités thermiques

$a_{\mathbf{i}}$ diffusivités

$h_{2}$ coefficient d'échange

$z, r$ variables spaciales

$t \quad$ variable temporelle

$T_{\mathrm{i}}$ températures.

Milieu 3. Cylindre échantillon. - Le problème correspondant au milieu 3 est représenté par le système :

$$
\frac{\partial^{2} T_{3}}{\partial z^{2}}+\frac{1}{r} \frac{\partial T_{3}}{\partial r}+\frac{\partial^{2} T_{3}}{\partial r^{2}}=\frac{1}{a_{3}} \frac{\partial T_{3}}{\partial t}, \text { avec les conditions aux limites : }
$$

aux paragraphes 2.2 et 2.3

$$
\begin{array}{lll}
\frac{\partial T_{3}\left(r,-l_{3}, t\right)}{\partial z}=-\frac{\phi(t)}{\lambda_{3}} & & \frac{\partial T_{3}\left(r,-l_{3}, t\right)}{\partial z}=0 \\
\frac{\partial T_{3}(r, 0, t)}{\partial z}=-\frac{\phi^{\prime}(t)}{\lambda_{3}} & \text { pour } 0 \leqslant r \leqslant r_{2} & \frac{\partial T_{3}(r, 0, t)}{\partial z}=\frac{\phi(t)-\phi^{\prime}(t)}{\lambda_{3}} \\
\frac{\partial T_{3}(r, 0, t)}{\partial z}=0 & \text { pour } r_{2}<r \leqslant r_{3} & \frac{\partial T_{3}(r, \partial, t)}{\partial z}=\frac{\phi(t)}{\lambda_{3}} \\
\frac{\partial T_{3}\left(r_{3}, z, t\right)}{\partial r}=0 & \text { pour }-l_{3} \leqslant z \leqslant 0 & \text { inchangé }
\end{array}
$$

au paragraphe 2.4 et la condition initiale

$$
T_{3}(r, z, 0)=0 \quad \forall r \quad \text { et } \quad \forall z \in \text { milieu } 3 .
$$

Equation de passage du milieu 3 au milieu 2.

On écrit que :

$$
\bar{T}_{3}-\bar{T}_{2}=r_{\mathrm{c}} \phi^{\prime}(t) \text { en } z=0,
$$

Milieu 2. Thermomètre. - On a :

$$
\frac{\partial^{2} T_{2}}{\partial z^{2}}+\frac{1}{r} \frac{\partial T_{2}}{\partial r}+\frac{\partial^{2} T_{2}}{\partial r^{2}}=\frac{1}{a_{2}} \frac{\partial T_{2}}{\partial t}
$$

expression dans laquelle $\bar{T}_{3}$ et $\bar{T}_{2}$ sont les températures moyennes, soient :

$$
\bar{T}_{3}=\frac{1}{\pi r_{2}^{2}} \int_{0}^{r_{3}} T_{3} 2 \pi r d r
$$

avec les conditions aux limites :

$$
\begin{gathered}
\frac{\partial T_{2}(r, 0, t)}{\partial z}=\frac{\phi^{\prime}(t)}{\lambda_{2}} \\
T_{2}\left(r, l_{2}, t\right)=0 \\
\lambda_{2} \frac{\partial T_{2}\left(r_{2}, z, t\right)}{\partial r}=-h_{2} T_{2}\left(r_{2}, z, t\right)
\end{gathered}
$$

et

$$
\bar{T}_{2}=\frac{1}{\pi r_{1}^{2}} \int_{0}^{r_{2}} T_{2} 2 \pi r \mathrm{~d} r
$$

et où $r_{\mathrm{c}}$ est la résistance de contact par unité de surface.

Cette équation de passage utilise des températures moyennes $\left(\phi^{\prime}(t)\right.$ est indépendant de $\left.r\right)$. Elle suppose aussi l'acceptation de l'extension de la notion de résistance de contact aux régimes transitoires.

$$
T_{2}(r, z, 0)=0 \quad \forall r, \forall z \in \text { milieu } 2 .
$$


En toute rigueur la notion de résistance thermique n'a de sens qu'en régime permanent; le déficit de matière au niveau de l'interface modifie l'inertie thermique $[18,19]$. De plus les dilatations provoquent un déplacement des zones en contact réel, de sorte que lorsqu'on utilise cette notion de régime variable on effectue une approximation. Il a été montré [20] que l'erreur alors commise reste faible.

La solution en $z=0$ est de la forme :

$$
\begin{aligned}
T_{3}= & \int_{0}^{t} \sum_{i=1}^{\infty} A_{i} \exp \left[-p_{i}(t-\tau)\right] \mathrm{d} \tau- \\
& -\int_{0}^{t} \phi^{\prime}(\tau) B\left\{1+2 \sum_{k=1}^{\infty} \exp \left[-u_{k}(t-\tau)\right]\right\} \cdots \\
& \ldots\left\{1+\sum_{n=1}^{\infty} C_{n} \exp \left[-v_{k}(t-\tau)\right]\right\} \mathrm{d} \tau \\
T_{2}= & \int_{0}^{t} \phi^{\prime}(\tau) \sum_{k=1}^{\infty} \sum_{n=1}^{\infty} D_{n} \exp \left[-\omega_{n k}(t-\tau)\right] \mathrm{d} \tau .
\end{aligned}
$$

En utilisant la condition de couplage thermique, on obtient une équation intégrale. La densité de flux $\phi^{\prime}(t)$ solution de cette équation intégrale est calculée numériquement. Ensuite on détermine $\bar{T}_{3}$ et $\bar{T}_{2}$ valeurs moyennes de $T_{3}$ et $T_{2}$ sur la surface de contact.

On suppose, comme en régime permanent, que la température mesurée est :

$$
\theta(t)=\frac{\lambda_{3} \bar{T}_{3}(t)+\lambda_{2} \bar{T}_{2}(t)}{\lambda_{3}+\lambda_{2}}
$$

Aux temps faibles il est intéressant de substituer à cette expression une expression semblable où interviennent les effusivités des matériaux. Ceci modifie peu les résultats et présente l'inconvénient de ne pas assurer le raccord avec le régime permanent aux temps longs.

Nous adopterons, comme en régime permanent, la définition suivante de l'erreur :

$$
\varepsilon=\frac{T_{3}^{\prime}-\theta}{T_{3}^{\prime}}
$$

où $T_{3}^{\prime}$ est la température du milieu 3 en $z=0$ en l'absence de détecteur, avec un flux $\Phi_{(t)}^{\prime}=\pi r_{2}^{2} \phi^{\prime}(t)$ uniformément réparti sur la face où auraient été appuyés les détecteurs :

$$
\begin{aligned}
T_{3}^{\prime}=\int_{0}^{t} \sum_{i=1}^{\infty} A_{i} \exp \left[p_{i}(t-\tau)\right] \mathrm{d} \tau- \\
\quad-\int_{0}^{t} \phi^{\prime}(\tau) B\left[1+2 \sum_{n=1}^{\infty}-u_{k}(t-\tau)\right] \mathrm{d} \tau .
\end{aligned}
$$

Le deuxième terme de cette expression était déjà dans l'expression de $T_{3}$. C'est ce qui permet d'éliminer des résultats, comme en permanent, l'influence de l'épaisseur du milieu 3.

2.2 MILIEU « ÉPAIS 》 RECEVANT UN DIRAC DE FLUX SUR LA FACE OPPOSÉE AU DÉTECTEUR. - A l'aide de ce modèle nous avons étudié théoriquement le comportement d'un matériau épais $\left(l_{3} \gg r_{1}\right)$.

Sur les figures 12 et 13 , nous avons tracé les variations de l'erreur $\varepsilon$ en fonction du temps pour un milieu conducteur et un milieu isolant (et avec diverses valeurs de $r_{2}$ et des résistances de contact), lorsque les détecteurs sont en $\mathrm{Bi}_{2} \mathrm{Te}_{3}$ à contacts séparés.

On observe :

- que l'erreur tend rapidement vers l'erreur en régime permanent, avant que ce régime permanent ne soit atteint,

- que l'erreur est constamment décroissante,

- pour les conducteurs $\left(\lambda_{3}=100 \mathrm{~W} / \mathrm{m}^{-1} \mathrm{~K}^{-1}\right)$ l'erreur est faible; la résistance de contact fait varier l'erreur dans des proportions importantes, le rayoǹ $r_{1}$ du détecteur intervient également;

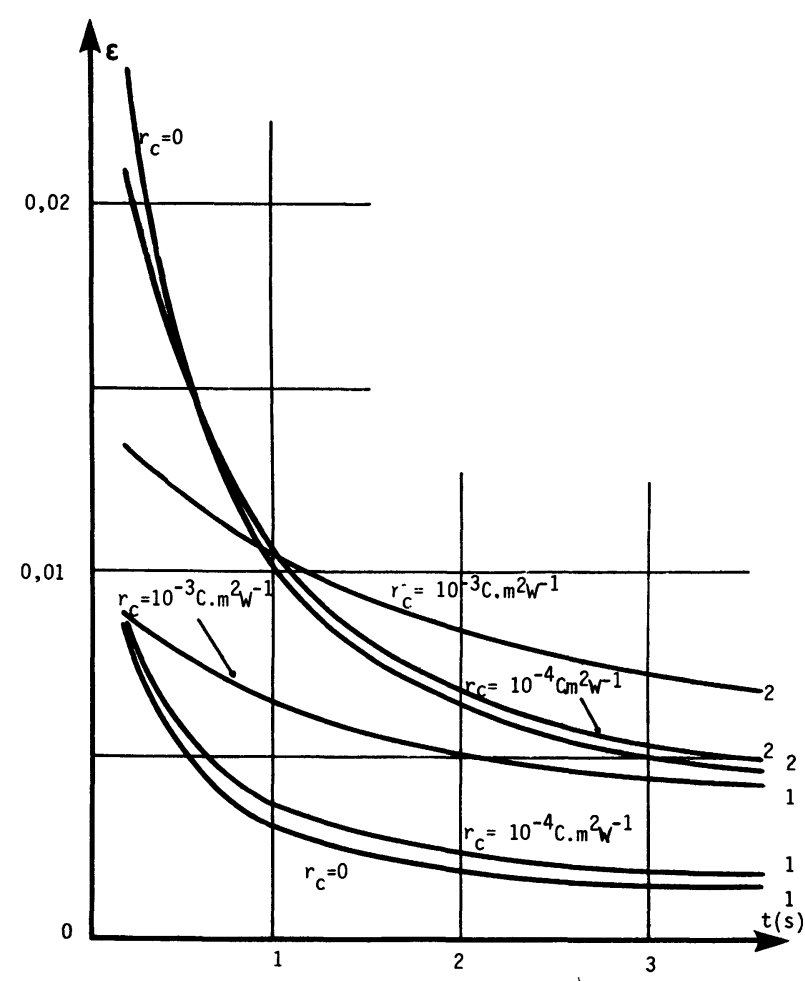

Fig. 12. - Fonctions erreur $\varepsilon$; Dirac face avant; contacts séparés; $r_{2}=1: 5 \times 10^{-4} \mathrm{~m}, 2: 2 \times 10^{-3} \mathrm{~m}$;

$$
\lambda_{3}=100 \mathrm{~W} \cdot \mathrm{m}^{-1} \cdot \mathrm{K}^{-1} ; \lambda_{2}=1 \mathrm{~W} \cdot \mathrm{m}^{-1} \cdot \mathrm{K}^{-1} ;
$$

$l_{3}=5 \times 10^{-3} \mathrm{~m} ; l_{2}=10^{-2} \mathrm{~m} ; a_{3}=0,2 \times 10^{-4} \mathrm{~m}^{2} . \mathrm{s}^{-1}$; $a_{2}=0,8 \times 10^{-6} \mathrm{~m}^{2} \cdot \mathrm{s}^{-1}$.

[Error functions $\varepsilon$; Dirac function (front surface); separated contacts : $r_{2}=1: 5 \times 10^{-4} \mathrm{~m}, 2: 2 \times 10^{-3} \mathrm{~m}$;

$$
\lambda_{3}=100 \mathrm{~W} \cdot \mathrm{m}^{-1} \cdot \mathrm{K}^{-1} ; \lambda_{2}=1 \mathrm{~W} \cdot \mathrm{m}^{-1} \cdot \mathrm{K}^{-1} ;
$$

$l_{3}=5 \times 10^{-3} \mathrm{~m} ; l_{2}=10^{-2} \mathrm{~m} ; a_{3}=0.2 \times 10^{-4} \mathrm{~m}^{2} . \mathrm{s}^{-1}$; $a_{2}=0.8 \times 10^{-6} \mathrm{~m}^{2} . \mathrm{s}^{-1}$.] 


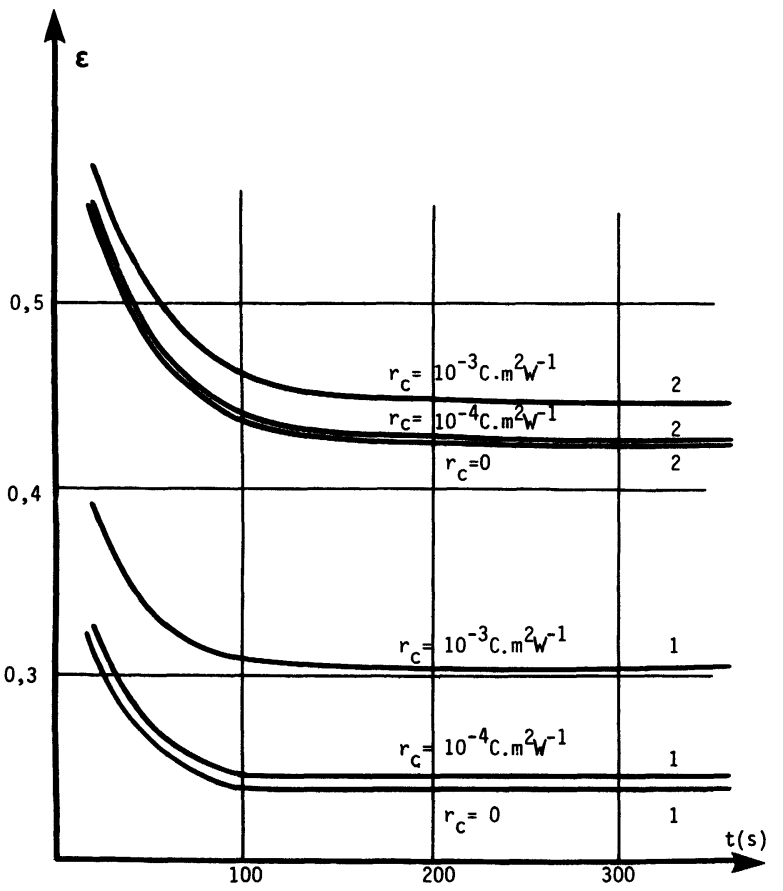

Fig. 13. - Fonctions erreur $\varepsilon$; Dirac face avant; contacts séparés; $r_{2}=1: 5 \times 10^{-4} \mathrm{~m}, 2: 2 \times 10^{-3} \mathrm{~m}$;

$$
\begin{gathered}
\lambda_{3}=0,2 \mathrm{~W} \cdot \mathrm{m}^{-1} \cdot \mathrm{K}^{-1} ; \quad \lambda_{2}=1 \mathrm{~W} \cdot \mathrm{m}^{-1} \cdot \mathrm{K}^{-1} ; \\
l_{3}=5 \times 10^{-3} \mathrm{~m} ; l_{2}=10^{-2} \mathrm{~m} ; r_{3}=24 \times 10^{-3} \mathrm{~m} ; \\
a_{3}=0,2 \times 10^{-6} \mathrm{~m}^{2} \cdot \mathrm{s}^{-1} ; a_{2}=0,8 \times 10^{-6} \mathrm{~m}^{2} \cdot \mathrm{s}^{-1} .
\end{gathered}
$$

[Error functions $\varepsilon$; Dirac function (front surface); separated contacts : $r_{2}=1: 5 \times 10^{-4} \mathrm{~m}, 2: 2 \times 10^{-3} \mathrm{~m}$;

$$
\begin{gathered}
\lambda_{3}=0.2 \mathrm{~W} \cdot \mathrm{m}^{-1} \cdot \mathrm{K}^{-1} ; \quad \lambda_{2}=1 \mathrm{~W} \cdot \mathrm{m}^{-1} \cdot \mathrm{K}^{-1} ; \\
l_{3}=5 \times 10^{-3} \mathrm{~m} ; \quad l_{2}=10^{-2} \mathrm{~m} ; r_{3}=24 \times 10^{-3} \mathrm{~m} ; \\
\left.a_{3}=0.2 \times 10^{-6} \mathrm{~m}^{2} . \mathrm{s}^{-1} ; \quad a_{2}=0.8 \times 10^{-6} \mathrm{~m}^{2} \cdot \mathrm{s}^{-1} .\right]
\end{gathered}
$$

- pour les milieux isolants, il faut supposer que les contacts électriques sont assurés par une couche conductrice d'épaisseur suffisamment faible pour être négligée dans l'analyse.

Cette fois la dimension de l'aire de contact $\left(\pi r_{2}^{2}\right)$ est prépondérante sur la valeur des erreurs $\varepsilon$, ceci peut être relié au rôle joué par la macroconstriction. Il apparaît nettement que la section des détecteurs doit être la plus faible possible.

Ces résultats sont semblables à ceux de Cassagne [5] avec la différence suivante : la résistance de contact intervient de façon moins sensible pour les milieux conducteurs; cela est dû aux contacts séparés.

Nous n'avons pas, pour ces milieux épais, tenté d'effectuer une étude expérimentale.

2.3 MILIEU « FIN 》 RECEVANT UN FLUX SUR LA FACE OPPOSÉE AUX DÉTECTEURS. - Certaines parties du dispositif expérimental utilisé pour cette étude sont identiques à celles du dispositif en régime permanent décrit dans la première partie (1.1).
Ce sont les systèmes d'application des forces et les détecteurs eux-mêmes. Les échantillons, la source de flux thermique et la chaîne d'acquisition de données sont différentes. Le schéma de principe de l'ensemble est représenté sur la figure 14.

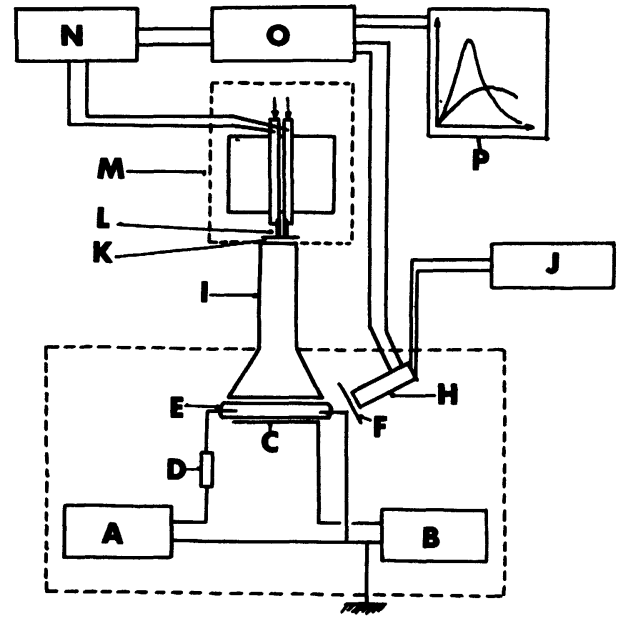

Fig. 14. - Schéma de l'installation expérimentale; A : alimentation flash; B : alimentation Trigger; C : Trigger; $\mathrm{D}$ : self; $\mathrm{E}$ : flash; $\mathrm{F}$ : filtres; $\mathrm{G}$ : «cage " métallique; $\mathrm{H}$ : photomultiplicateur ; I : fibres optiques; $\mathbf{J}$ : alimentation $\mathbf{P M} ; \mathbf{K}$ : échantillon; $\mathbf{L}$ : détecteurs; $\mathbf{M}$ : enceinte; $\mathbf{N}$ : amplificateur; $\mathrm{O}$ : enregistreur à mémoires; $\mathrm{P}:$ enregistreur potentiométrique.

[Sketch of the experimental apparatus. A : flash supply; B : trigger supply; C : trigger ; D : coil ; E : flash ; F : filters; G : metallic cage; $H$ : photomultiplier; I : optical fibers; J : photomultiplier supply; $\mathrm{K}$ : sample; $\mathrm{L}$ : thermoelectric elements; $\mathbf{M}$ : housing; $\mathbf{N}$ : amplifier ; $\mathrm{O}$ : transient recorder; $P$ : potentiometric recorder.]

L'éclair de 450 ou $830 \mu$ s de durée, fourni par un tube "flash", est conduit par un faisceau de fibres optiques sur l'échantillon et provoque son échauffement.

La forme de cet éclair en fonction du temps est relevée à l'aide d'un photomultiplicateur. Nous nous sommes assurés, en comparant les courbes de transmission en fonction de la longueur d'onde des fibres optiques, des atténuateurs, des milieux transparents, et la courbe de sensibilité du photomultiplicateur que la forme restituée par le photomultiplicateur était bien celle absorbée par l'échantillon. Le signal est stocké dans un enregistreur à mémoires, puis restitué plus lentement sur un enregistreur potentiométrique. La f.é.m. délivrée par le thermocouple est amplifiée, puis traitée de la même façon que le signal issu du photomultiplicateur.

Nous donnerons les résultats en comparant les évolutions expérimentales des températures à celles calculées en l'absence de détecteurs (Fig. 15) et aux résultats du modèle (Figs. 16 et 17) pour des contacts séparés et uniques. Connaissant le gain de l'ampli- 
ficateur ( $\times 100)$, la sensibilité de chaque enregistrement et le pouvoir thermoélectrique des couples, nous déterminons un ordre de grandeur de l'élévation de température mesurée par ceux-ci. Elle est d'environ $10^{\circ} \mathrm{C}$. Cependant, ne pouvant chiffrer avec précision l'énergie absorbée par la face avant, nous avons normalisé ces courbes par rapport au maximum atteint.

On observe que :

- Le modèle conduit à une réponse quasiment parfaite pour les contacts séparés alors que le thermomètre en contact unique présente un retard appréciable;

- La courbe expérimentale à contacts séparés ne correspond pas exactement au modèle, elle est " en retard ». Ceci doit être attribué aux conditions de contact notamment aux traces de $\mathrm{Bi}_{2} \mathrm{Te}_{3}$ déposées sur le cuivre.

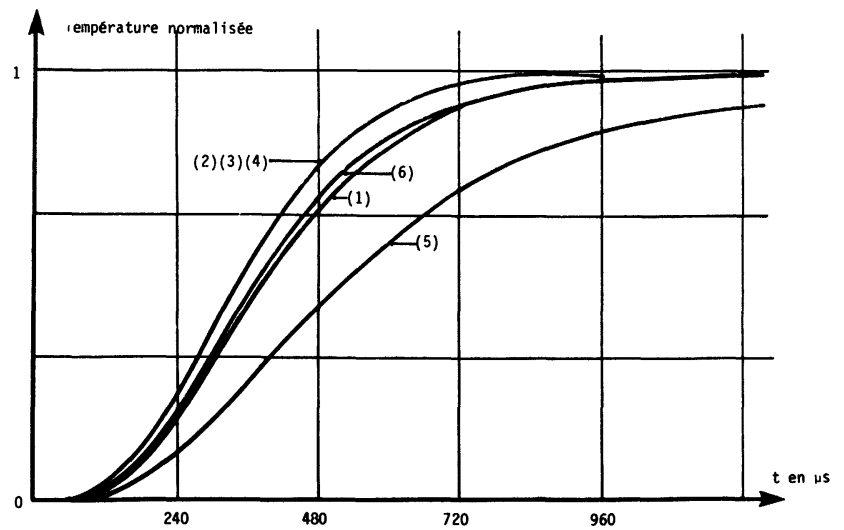

Fig. 15. - Courbes de températures normalisées :

1 : Réponse expérimentale. Echantillon en cuivre. Force d'appui $1 \mathrm{~N}$.

2 : Température calculée en l'absence de détecteurs.

Contacts 3 : Simulation : température mesurée par le séparés couple avec $r_{\mathrm{c}}=10^{-4} \mathrm{~K} \cdot \mathrm{m}^{2} . \mathrm{W}^{-1}$

4 : Simulation : température mesurée par le couple avec $r_{\mathrm{c}}=10^{-5} \mathrm{~K} \cdot \mathrm{m}^{2} \cdot \mathrm{W}^{-1}$.

6 : Réponse expérimentale. Echantillon en cuivre. Force d'appui 3,5 N.

Contact $\{5:$ Simulation : température mesurée par le

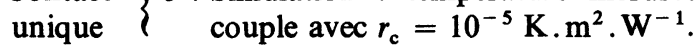

[Normalized temperature curves (long flash) :

1 : Experimental response (copper sample; load $1 \mathrm{~N}$ ).

2 : Computed temperature without the thermoelectric elements.

Separated $3:$ Simulation : measured temperature with

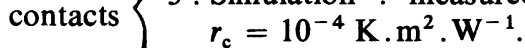

$4:$ Simulation : measured temperature with $r_{\mathrm{c}}=10^{-5} \mathrm{~K} \cdot \mathrm{m}^{2} \cdot \mathrm{W}^{-1}$

6 : Experimental response (copper sample; load $3.5 \mathrm{~N})$.

Single $\{5:$ Simulation : measured temperature with contact $\left\{\begin{array}{r}5 \\ r_{\mathrm{c}}=10^{-5} \mathrm{~K} \cdot \mathrm{m}^{2} \cdot \mathrm{W}^{-1} \text {.] }\end{array}\right.$

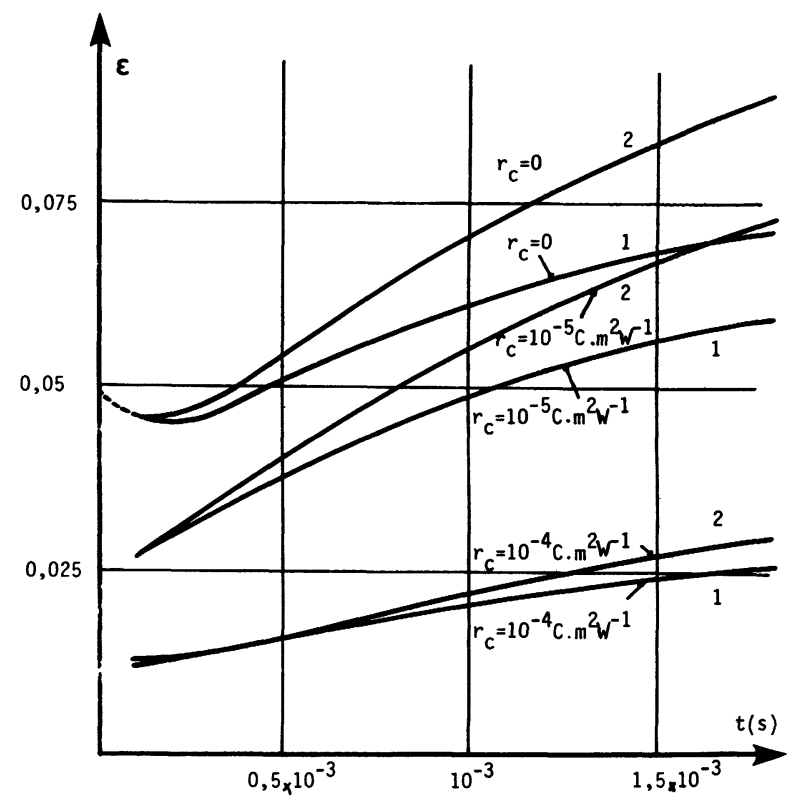

Fig. 16. - Fonctions erreur $\varepsilon$; Dirac de flux sur la face avant ; thermocouple à contacts séparés; $r_{2}=1: 5 \times 10^{-4} \mathrm{~m}$, $2: 2 \times 10^{-3} \mathrm{~m} ; r_{3}=2,4 \times 10^{-2} \mathrm{~m} ; \lambda_{3}=100 \mathrm{~W} \cdot \mathrm{m}^{-1} \cdot \mathrm{K}^{-1}$; $\lambda_{2}=1 \mathrm{~W} \cdot \mathrm{m}^{-1} \cdot \mathrm{K}^{-1} ; l_{3}=10^{-4} \mathrm{~m} ; l_{2}=10^{-2} \mathrm{~m} ; a_{3}=$ $0,2 \times 10^{-4} \mathrm{~m}^{2} \cdot \mathrm{s}^{-1} ; a_{2}=0,8 \times 10^{-6} \mathrm{~m}^{2} \cdot \mathrm{s}^{-1}$.

[Error functions $\varepsilon$, Dirac function (front surface); separated contacts; $r_{2}=1: 5 \times 10^{-4} \mathrm{~m}, 2: 2 \times 10^{-3} \mathrm{~m} ; r_{3}=2.4 \times$ $10^{-2} \mathrm{~m} ; \lambda_{3}=100 \mathrm{~W} \cdot \mathrm{m}^{-1} \cdot \mathrm{K}^{-1} ; \lambda_{2}=1 \mathrm{~W} \cdot \mathrm{m}^{-1} \cdot \mathrm{K}^{-1}$; $l_{3}=10^{-4} \mathrm{~m} ; l_{2}=10^{-2} \mathrm{~m} ; a_{3}=0.2 \times 10^{-4} \mathrm{~m}^{2} \cdot \mathrm{s}^{-1}$; $a_{2}=0.8 \times 10^{-6} \mathrm{~m}^{2} \cdot \mathrm{s}^{-1}$.]

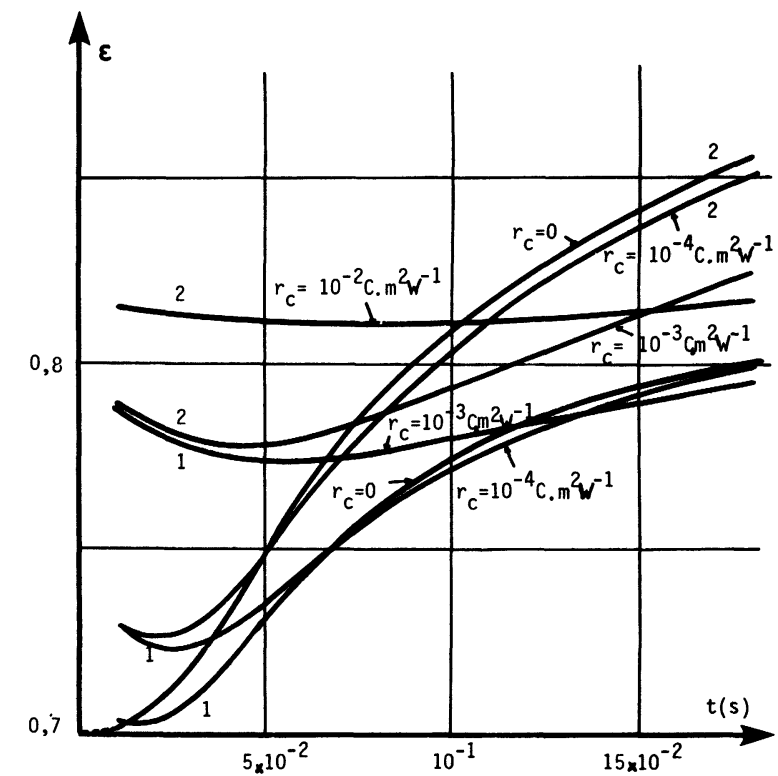

Fig. 17. - Fonctions erreur $\varepsilon$; Dirac de flux sur la face avant ; thermocouple à contacts séparés ; $r_{2}=1: 5 \times 10^{-4} \mathrm{~m}$, $2: 2 \times 10^{-3} \mathrm{~m} ; r_{3}=2,4 \times 10^{-2} \mathrm{~m} ; \lambda_{3}=0,2 \mathrm{~W} \cdot \mathrm{m}^{-1} \cdot \mathrm{K}^{-1}$; $\lambda_{2}=1 \mathrm{~W} \cdot \mathrm{m}^{-1} \cdot \mathrm{K}^{-1} ; l_{3}=10^{-4} \mathrm{~m} ; l_{2}=10^{-2} \mathrm{~m} ; a_{3}=$ $0,2 \times 10^{-6} \mathrm{~m}^{2} \cdot \mathrm{s}^{-1} ; a_{2}=0,8 \times 10^{-6} \mathrm{~m}^{2} \cdot \mathrm{s}^{-1}$.

[Error functions $\varepsilon$, Dirac function (front surface); separated contacts $; r_{2}=1: 5 \times 10^{-4} \mathrm{~m}, 2: 2 \times 10^{-3} \mathrm{~m} ; r_{3}=2.4 \times$ $10^{-2} \mathrm{~m} ; \lambda_{3}=0.2 \mathrm{~W} \cdot \mathrm{m}^{-1} \cdot \mathrm{K}^{-1} ; \lambda_{2}=1 \mathrm{~W} \cdot \mathrm{m}^{-1} \cdot \mathrm{K}^{-1}$; $l_{3}=10^{-4} \mathrm{~m} ; l_{2}=10^{-2} \mathrm{~m} ; a_{3}=0.2 \times 10^{-6} \mathrm{~m}^{2} \cdot \mathrm{s}^{-1}$; $a_{2}=0.8 \times 10^{-6} \mathrm{~m}^{2} \cdot \mathrm{s}^{-1}$.] 
Ces résultats montrent l'aptitude des détecteurs thermoélectriques semi-conducteurs, à contacts séparés à suivre des variations rapides de température de surface (retard largement inférieur à $100 \mu \mathrm{s}$ ).

L'ordre de grandeur des temps mesurés est assez inhabituel en thermique. Cependant il reste dans le domaine de validité de l'équation de Fourrier. Cette équation ne doit être modifiée que lorsque les durées sont de l'ordre de grandeur des temps de relaxation : $10^{-11} \mathrm{~s}$ [21].

L'erreur $\varepsilon$ pour un Dirac de flux reçu en $z=-l_{3}$ par des matériaux fins en fonction du temps présente la particularité de croître en fonction du temps. L'erreur pour les temps courts tend vers une valeur correspondant à une température en $z=0$ qui est dans le rapport des effusivités lorsque $r_{\mathrm{c}}=0$.

$$
\varepsilon=\frac{\sqrt{\rho_{2} c_{2} \lambda_{2}}}{\sqrt{\rho_{2} c_{2} \lambda_{2}}+\sqrt{\rho_{3} c_{3} \lambda_{3}}} .
$$

Pour les temps longs, l'erreur tend évidemment vers l'erreur du permanent.

2.4 MILIEU « ÉPAIS " RECEVANT UN FLUX SUR LA FACE OÙ SONT APPUYÉS LES DÉTECTEURS. - Le milieu est un cylindre transparent et une fine couche métallique $0,1 \mu \mathrm{m}$ (or) est vaporisée sur l'une de ses faces. Cet échantillon est placé dans le dispositif décrit au paragraphe précédent.

L'éclair lumineux traverse l'échantillon et vient échauffer la couche d'or qui est en contact avec les détecteurs en $\mathrm{Bi}_{2} \mathrm{Te}_{3}$. L'absorption dans le milieu transparent du flash incident est négligeable compte tenu de l'épaisseur des échantillons, et de leur transparence au rayonnement utilisé : en revanche dans l'infrarouge lointain émis par les diverses surfaces à la température d'expérimentation (ambiante), l'échantillon est opaque.

Au maximum, la température de la couche déposée $s^{\prime}$ élève de $5^{\circ} \mathrm{C}$. Cette fois le transfert de chaleur s'effectue de la surface $z=0$ vers l'intérieur du milieu. Sur la figure 18 ci-après, sont reportées les évolutions de température normalisées à 1 :

- du matériau en l'absence de détecteurs,

- celles obtenues théoriquement et expérimentalement pour un thermomètre à contacts séparés,

- et celle obtenue pour un thermomètre à contact unique.

Les résultats sont très semblables à ceux provenant de la mince feuille de cuivre.

Les ordres de grandeur des temps de "retard" sont les mêmes et la réponse expérimentale est encore plus lente que celles prévues par le modèle. Les conditions de contact en sont, ici, encore la cause.

Il apparaît dès maintenant qu'il sera extrêmement difficile d'élaborer un modèle prenant en compte exactement ces conditions de contact. En effet, la métrologie à mettre en ouvre se situe au niveau de la microgéométrie des contacts eux-mêmes; les pro-

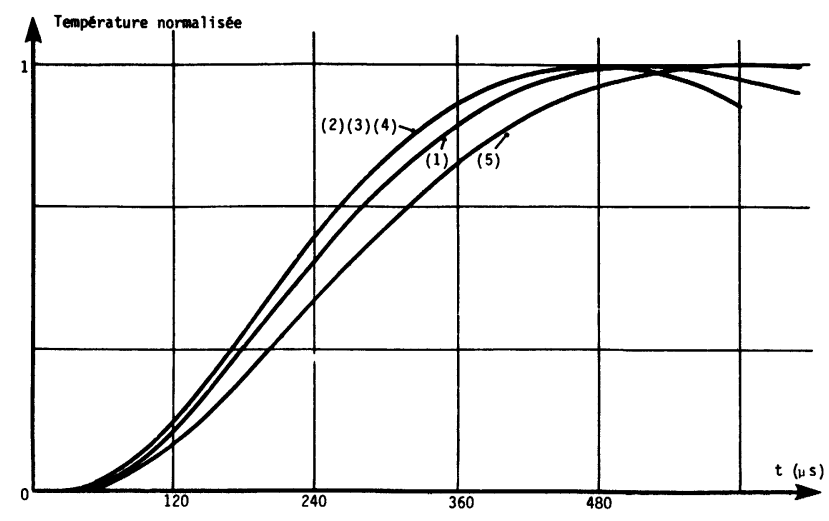

Fig. 18. - Courbes de températures normalisées.

$$
\begin{aligned}
& \text { ( } 1 \text { : Réponse expérimentale. Echantillon en } \\
& \text { Puropsil (quartz naturel). }
\end{aligned}
$$

Contact $\{5:$ Simulation : température mesurée par le unique $\left\{\right.$ couple si $r_{\mathrm{c}}=10^{-4} \mathrm{~K} \cdot \mathrm{m}^{2} \cdot \mathrm{W}^{-1}$.

[Normalized temperature curves (long flash) :

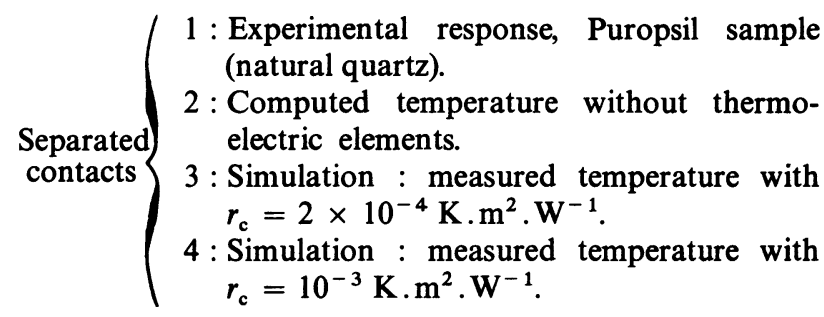

Single $\{5:$ Simulation : measured temperature with contact $\left\{\begin{array}{r}r_{\mathrm{c}}=10^{-4} \mathrm{~K} \cdot \mathrm{m}^{2} \cdot \mathrm{W}^{-1} \text {.] } \\ \text {. }\end{array}\right.$

priétés thermophysiques des matériaux doivent être connues immédiatement sous la surface; les calculs sont excessivement complexes.

3. Conclusion. - Les thermocouples de surface à contacts séparés en tellurure de bismuth possèdent des qualités très intéressantes aussi bien en régime permanent qu'en régime transitoire; elles proviennent de leur faible conductivité thermique : $1 \mathrm{~W} / \mathrm{m}^{-1} \mathrm{~K}^{-1}$.

Ils peuvent être utilisés à des très basses températures et, de plus, ils possèdent une sensibilité, environ dix fois plus grande $(350 \mu \mathrm{V} / \mathrm{K}$ à $290 \mathrm{~K})$ que les thermocouples classiques.

D'autres matériaux semiconducteurs peuvent être utilisés pour des applications à de plus hautes températures, par exemple les alliages Ge-Si jusqu'à $1100 \mathrm{~K}$. Des problèmes d'oxydation et de diffusion rendent leur emploi plus délicat. De façon générale tous les matériaux qui ont été développés pour la conversion thermoélectrique de la chaleur en électricité seront bien adaptés aux mesures de température de surface puisqu'ils ont été étudiés pour avoir un facteur de mérite élevé, c'est-à-dire une faible conductivité thermique et un fort pouvoir thermoélectrique. 


\section{Bibliographie}

[1] Henning, C. D., Parker, R., Transient response of an intrinsic thermocouple. J. Heat Trans. 89 (1967) 146-154.

[2] Poncin, H., Les apports de la thermocinétique à la détermination des températures de surface. Cahiers de la thermique, no 3, Série A (1973).

[3] Satyamurthy, P., Marwah, R. K., Venkatramani, N. et Rohatgi, V. K., Int. J. Heat Mass Trans. 22 (1979) $1151-1154$.

[4] Bardon, J. P., La mesure des températures de surface par contact. Erreurs liées aux transferts de chaleur parasites. Rev. Gen. Therm. (France) 15 (1976) 121.

[5] Cassagne, B., Kirsch, G. et Bardon, J. P., Analyse théorique des erreurs liées aux transferts de chaleur parasites lors de la mesure d'une température de surface par contact. Int. J. Heat Mass Transfer. 23 (1980) 1207-1217.

[6] Degiovanni, A., Gery, A., Laurent, M., Macqueron, J. L. et Sinicki, G., C.R. Hebd. Séan. Acad. Sci. Paris 280 (1975) 393.

[7] Gery, A., Laurent, M., Sinicki, G. et Degiovanni, A., Entropie no 70 (1976) 31.

[8] Gery, A., Thèse Doctorat d'Etat, Lyon 1980.

[9] BRUN, M. J., Mesure des températures de surface. Publ. no 54, Edit. Europ. Techn. et Indust., janvier 1976.

[10] Donaldson, I. S. et Haslett, R. A., A fast response surface thermocouple. J. Mech. Eng. 16 (1974) 174-177.

[11] HuETz, J., Evolution rapide des températures de surface. Rev. Gén. Electr. 79 (1970) 833-837.

[12] Boyer, J., Cassien, M., Huetz, J., Petit, J. P., Nouvelles techniques thermoélectriques de mesure de température, $4^{\mathrm{e}}$ conf. Int. de Heat Transfer, Versailles 1970.
[13] Rogoff, G. L., Thermocouple structure for measurements of surface temperature. Rev. Sci. Instrum. (USA) 44 (1973) 654.

[14] BAILEY, W. J., The correct way to contact surface temperature measurement. Control. Eng. (USA) 23 (1976) 73-75.

[15] PaK, V. et Kalinin, A. N., Precision measurement of steady surface temperatures with a contact thermometer. Ind. Lab. (USA) 42 (1976) 1747.

[16] Bouisson, H., Influence, lors de mesures de températures de surface à l'aide de thermocouples, du rayonnement reçu par ceux-ci et de leur mode de fixation. Ann. Phys. no 7 (1962) 535.

[17] Cassagne, B., Contribution à la mesure des températures de surface par contact. Application au contrôle non destructif. Thèse $3 \mathrm{e}$ cycle, Nantes 1974.

[18] Saint Blanquet, C., Etude du transfert de chaleur entre solides accolés en régime thermique sinusoïdal. Thèse Nantes 1970.

[19] Vullierme, J. J., Transfert de chaleur non permanent " modélisation de l'interface ". Journées d'Etudes de la Soc. Française des Thermiciens sur les résistances thermiques de contact. Paris le 12 décembre 1979.

[20] SiNICKI, J. G., Contribution à la mesure des résistances thermiques en régime transitoire ". Thèse juin 1975, Lyon.

[21] Domanski, R., "Establishing application limits for classical Fourrier on wave heat conduction equations for metals on the basis of analytical solution and calculated temperature distribution in case of giant heat pulse " 6th Conf. Int. of Heat Transfer, Toronto, août 1978. 